\title{
The Role of Emotionality and Power on Tendency to Unethical Behaviors
}

\author{
Hossein Safarzadeh \\ Department of Business Administration, Central Tehran Branch, Islamic Azad University, \\ Tehran, Iran \\ Email: hr.safarzadeh@gmail.com
}

\begin{abstract}
Alireza Soloukdar
(Corresponding author)

Department of Ind ustrial Management, Central Tehran Branch, Islamic Azad University, Tehran, Iran

Email: a_soloukdar@ hotmail.com
\end{abstract}

\begin{abstract}
Ali Alipour
MA in Human Resource Management, University of Tehran, School of Business, Iran Email: ali.alipour@ut.ac.ir

Seyedeh Akram Parpanchi

Department of Information Technology Management, Electronic Branch, Islamic Azad University, Tehran, Iran

Email: akramparpanchi@ hotmail.com
\end{abstract}

Accepted: November 02, 2012 Published: December 14, 2012

Doi:10.5296/ijhrs.v2i4.2866 URL: http://dx.doi.org/10.5296/ijhrs.v2i4.2866

\begin{abstract}
Emotion and power have been studied very deeply especially during the last decade; however, the common effects of these two factors on individuals' optional behaviors (organizational citizenship, anti-productivity, unethical behaviors) have been less focused. In the present paper, the role of individuals' emotionality, their interest in power, and their tendency to unethical behaviors will be discussed according to the model of Levine. Three questionnaires were distributed and the collected data were analyzed using Pearson's Correlation Coefficient, Multiple Regression, and T- test. Findings indicated that there is a
\end{abstract}


significant relationship between the two independent variables of emotionality and power and tendency to unethical behaviors. Moreover, according to the results of the tests, men are more emotion-oriented and power seekers than women.

Keywords: Power, Emotion, Ethical Behaviors

\section{Introduction}

Investigation on Arthur Anderson Company's bankruptcy, which was a reputable company in the past, indicated the effects of illegal and ethically-questioned catastrophic counter-productivity behaviors in the personal and also organizational levels as the cause of loss of billions of dollars, dismissing of many employees and shareholders and a big bankruptcy over a period of time (Levine, 2010). This example and many others show the effects of unethical behaviors on organization success or failure. Ethical issues related to job environment have been increased and focused more during the last recent years. Having known the growth of ITC networks and the related speed, the need for ethical behaviors is inevitable. People's judgment about good and bad things is as important as their performance in information productivity and transmission. This judgment is affected by many parameters. From Naude's perspective, these parameters are classified in three main levels presented in the next paragraph.

In macro level, such factors as cultural variety, economic conditions, technology, religion and laws are the examples of environmental factors. In the middle level, factors like competition, reward system, behavioral principles, job features, organizational culture, objectives, organizational environment, position seeking, performance evaluation and the reference position are included. And at the micro level, individual factors as control, self-power, attitude, value, belief, perception, and demography are discussed (Naude, 2004).

It is clear that individual differences have a great effect on their behavior. During the recent years, a lot of attention has been given to the relationship between employees' behavior and organizational and occupational outcomes. After the emergence of psychological revolution, a new tendency to management studies has arisen with the focus on emotionality and personal characteristics and their effects on behavior in a way that emotion and power have been addressed as the parts of organizational culture, and now we can see the examples of power-oriented organizations, competitive organizations, and busy organizations in organizational classification (Rafaeil Sutton, 1989).

Sackett and Devore (2001) sated that losses and disadvantages of unethical behaviors will affect both employees and the organization itself. Such behaviors include abuse of authority, lying and cheating in business transactions, using individuals to serve the organization while they are not satisfied, unfair rewards, marketing defective goods, and etc.

Several researches have been conducted about the relationship between personality characteristics and ethical behavior in organizations; one of the examples of these researches is the study of Brown et al. (2010), in which they found that personality characteristics of empathy and egoism will lead to ethical decisions. In another study, Rallapally at al. (1994) investigated the relationship between ethical beliefs and personality characteristics and 
concluded that people who are independent, innovative, and aggressive as well as those with higher degrees of risk, are less adherent to ethics, and those people seeking problem solving are more adherent.

\section{Influencing Factors}

\subsection{Emotion and Emotionality}

Emotions can be interpreted as the most comprehensive expressions for a group of events including feelings and feelings states, and as a complicated state of an individual or a social system including some different but related components namely feelings associated with experience, expressing feelings using verbal and non-verbal signals, physiological states, perception, functional techniques and behaviors (Russell et al., 2003).

The term "Emotion" is rooted in the Latin word of "Emovere" which means movement, motivation, and tension state. Emotion is equal to the start of passivity feeling and kindness. Emotion is a complicated psychological state including three separate factors of mental experience, emotional response, and the obvious behavior resulting from this experience (Atkinson et al., 2006).

Emotion is the complex response of an individual out of an evaluation of interactions associated with the self and environment, and it is derived from the emotional state, direction of attention, facial expression, performance, and behavior (Levine, 2010). Izard (1993) defines the emotion as a response including the interaction between cognitive, physiological, communicative, and functional factors which help to adapt with those opportunities and challenges we face in important life events.

Many managers recognize the power of emotion in the organization and are seeking its benefits for the organization. Managers who understand the role of emotions will be more able to explain and predict the behavior of individuals. In general, if emotions are not identified, job performance will be faced with a serious obstacle (Ghasemi, 2010).

In understanding emotion and its effects on behavior, individuals' perceptions and cognition play a vital role; that is, individuals' behavior is deeply rooted in their perceptions and cognitions of their environment. Regarding this, Shaw (2003) claimed that every emotion includes cognitions, actions, and feelings, and each person can have different emotional reactions in the same position relative to the other persons'. In fact, this is a personal attribute that we can name emotionality.

Emotionality can be interpreted as the level of flexibility and emotion tolerance against happenings and events, a part of which is innate and resulted from hereditary characteristics and another part derived out of epistemology and self experiences (Clark, 1991). Emotionality has a special position in studies about emotional aspects in organizations, and its effect on other organizational structures has been approved. For example, in his study, Judge (1993) approved the close relationship between emotionality and such factors as social support, emotional exhaustion, depersonalization, and stress. Witt et al. (2002) also defined individuals' emotionality as an important factor for predicting the organizational commitment 
of staff. Rezaeeyan and Naeeji (2010) have focused on the important role of emotionality and personality of employees that can affect job satisfaction.

\subsection{Emotionality Dimensions}

Emotionality has two independent dimensions: Positive Attitude (PA) and Negative Attitude (NA). Negative attitude (NA) is a personal characteristic in which people are forced into a passive and negative reactions and has a close relationship with anxiety (Parkers, 1990). The other attitude is Positive Attitude (PA) which is developed after NA and shows a more optimistic interpretation of the phenomena in a way that individual shows a positive reaction (Spector et al, 2000). Gaudin and Thorne (2001), as an example, have studied the effect of emotion on individual ethical decisions and concluded that emotional state can affect individuals' tendency to unethical behaviors in a way that interpretations and judgments of a person will develop along with the improvement of emotions and will lead to upgrade in his/her ethical decisions. In another study conducted by Naquin and Holton (2002), the relationship between emotionality, personality traits, and commitment (including work ethics, emotional commitment, and emotional involvement) and motivation was investigated, in which positive attitude, commitment, and extroversion constituted 75 percent of the variance of motivation.

\subsection{Tendency to Power}

Power seeking was first introduced by Adorno and his colleagues (1950) in their book as Authoritarian Personality (TAP) in 9 classes of behaviors: "conventionalism, authoritarian submission, authoritarian aggression, anti-intraception, superstition and stereotype, power and toughness, destructiveness and cynicism, projectivity, and exaggerated concerns over sex".

Power is defined as a personality pattern that reflects the desire for security, order, power, and position in Heritage Dictionary, and also as a tendency to restructuring the power lines, a set of values and views, the demand for obedience and unquestionable desire to shield minorities or groups hostile to people and the use of non-traditional. It can be said that power is the probability to impose someone's will despite resistance in social relations (Weber, 1947).

Referring to Mintzberg, Parsaaeeyan (2009) stated that basically everyone is thirsty of power, and exerting an influence will depend on the following three conditions: 1) Having a source of power, 2) using the energy when needed, 3 ) in a way that is politically useful.

In Robbins' point of view, the main features of personality affecting organizational behavior include control center, power or authority-orientation, self-esteem, and cope with the situation (Robins, 2009). Authoritarian personality was first introduced in social dominance theory in which the main variable is the difference between individuals in their social dominance (Sidanuse \& Paratto, 1999).

The authoritarian personality does not accept any command but orders. People with this personality type are seeking security, compliance, and stability. When they are facing 
uncomfortable events or situations, they feel anxious and not secured. They are fanatic to divergence of the things that they are committed to such as religion, race, nationality, history, culture, language, and etc (Adorno et al, 1950).

In a study conducted by Lawe et al. (2001), it was concluded that the power-oriented behavior of physicians in practice has led to inappropriate behavior.

Individuals with a high degree of this personality factor are seeking to overcome this factor as the main cause of conflict of human life (Kennedy, 2009).

In their study about the effect of authoritarian personality and age of police officers, Laguna et al. (2009), found that there is a significant relationship between this characteristic and anti-social behaviors.

According to the previous studies, and Levine's study about the effect of emotional power on counter-productive work behavior and organizational behavior for example, in this paper we have studied the effect of individuals' excitement and power-seeking on their tendency towards unethical behavior based on above mentioned background and tacit knowledge available at the micro level.

\section{Research Hypotheses}

Based on above mentioned background and tacit knowledge obtained, the following hypotheses were formulated:

H1: There is a significant relationship between emotionality and individuals' tendency to power.

$\mathrm{H} 2$ : There is a significant relationship between emotionality and unethical behaviors.

H3: There is a significant relationship between individuals' tendency to power and unethical behaviors.

H4: There is a significant relationship between gender and emotionality.

H5: There is a significant relationship between gender and individuals' tendency to power.

\section{Research Conceptual Model}

Considering the above mentioned hypotheses and with regard to Levine's model, the conceptual model of this research was developed as shown in figure 1. In the conceptual model, gender, emotionality, and power seeking are considered independent variables of the research, and unethical behavior is the dependent variable. However, it should be noted that since emotionality and tendency to power or power seeking are influenced by gender and impact on unethical behavior, they (emotionality and tendency to power) can also be treated as the intervening variables. Generally, the model presents five main relationships including: a relationship between emotionality and unethical behavior, a relationship between individuals' tendency to power and unethical behavior, a relationship between gender and 
tendency to power, a relationship between gender and emotionality, and a mutual relationship between emotionality and individuals' power seeking.

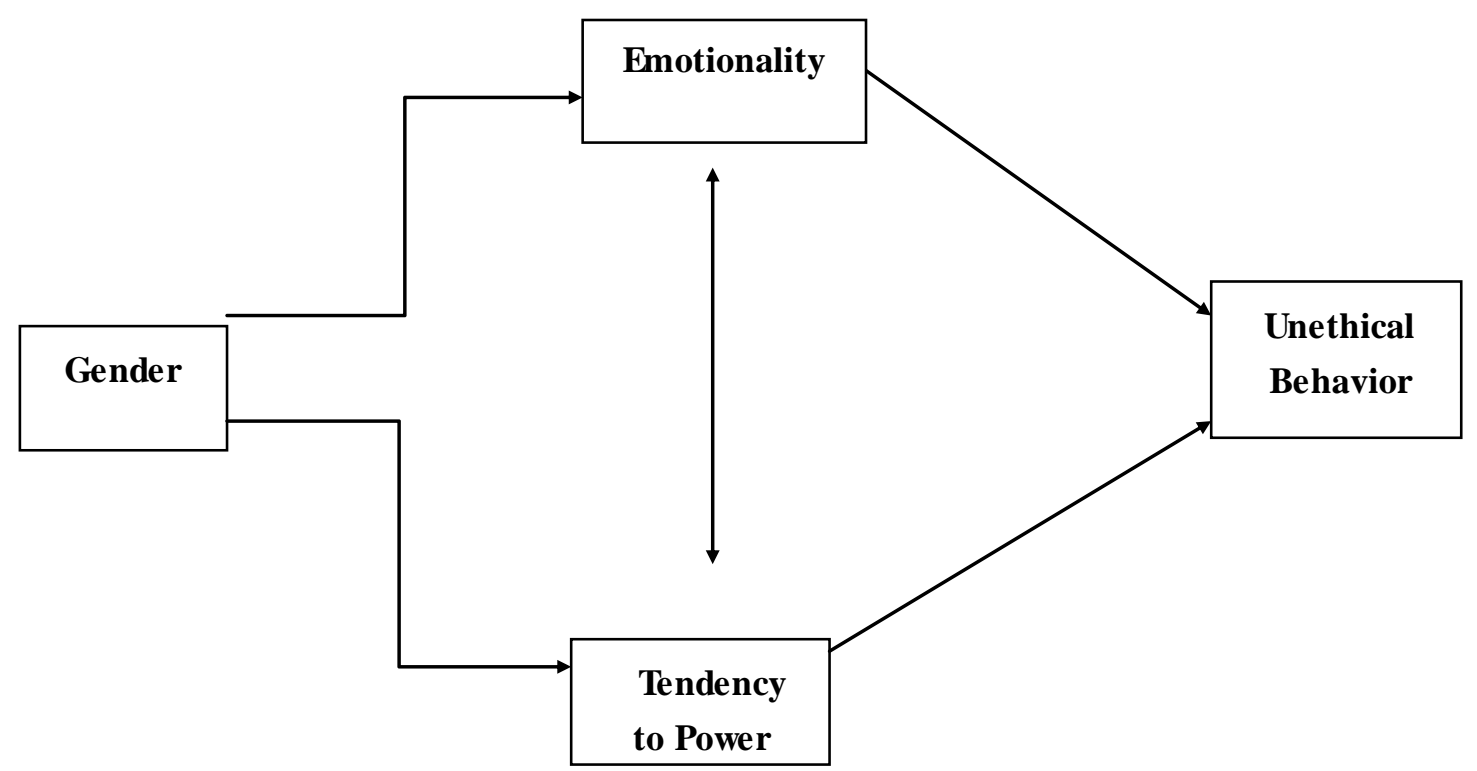

Figure 1: Research Conceptual Model

\section{Method}

\subsection{Sample Size and Sampling}

The current research is a practical study, and the method of data collection is descriptive survey. The study population included all workers in Iran's official gas company who are 419 persons. Sample size that was obtained through the Kerjesy and Morgan table added up to 201 individuals; simple random sampling method was used for selecting the samples.

\subsection{Research Tools}

Three survey questionnaires were used to collect data: 1. Emotionality questionnaire, 2. Power Measurement Questionnaire, and 3. Unethical behavior questionnaire.

\subsubsection{Emotionality Questionnaire}

Zuckerman scale was used to measure populations` emotionality degrees. The questionnaire contained 14 items and highly correlated with Jeffrey Arnet's emotional scale questionnaire. In the current study the reliability of this scale was calculated as $93 \%$ using Cronbach's alpha.

\subsubsection{Power Measurement Questionnaire}

This questionnaire included 10 items that were designed in 1970 by Christie and Jesse, and using a Likert range of five options measures people's tendency to power. The reliability of 
this scale was calculated as 79\% using Cronbach's alpha.

\subsubsection{Unethical Behavior Questionnaire}

This 15 items questionnaire was designed in 1993 by Louzyr. Cronbach's alpha coefficient calculated for this questionnaire was 0.9 which proves it to be appropriate.

\section{Results}

Research data were analyzed using Pearson's Correlation and Multiple Regression Analysis. For the first hypothesis, according to a distance of two variables, Simple Regression was used; based on the results, Pearson's correlation value equaled 0.183 which meant that the variable has the correlation of about 3.18 percent with tendency to power. $\mathrm{T}$ value and significance level of 0.001 concluded that the relationship was significant in 99 percent confidence interval; therefore the first hypothesis is confirmed.

About the second hypothesis, based on Pearson's correlation value of 0.181 , emotionality correlated with unethical behavior by 18.1 percent. The coefficient suggested that emotionality defines the variance of the unethical behavior by 3.3 percent. Significance levels of $\mathrm{T}$ and its amount of 3.442 and 0.001 were significant and showed that the relationship at confidence interval of 99 percent was significant. Therefore, the hypothesis is confirmed.

Regarding the third hypothesis, the Pearson's Correlation equaled 0.249 indicating that tendency to power as the independent variable had a correlation of 24.9 percent with unethical behavior. The coefficient suggested that the variable tendency to Power defines the variance of the unethical behavior as 6.2 percent. The value of $T$ and the significance levels of 4.802 and 0.000 respectively showed a significant relationship in 99 percent confidence interval, so this hypothesis is also confirmed.Table 1 shows the results of simple regression analysis.

Table 1: Regression Analysis between Emotionality and Power

\begin{tabular}{|c|c|c|c|c|c|c|c|c|}
\hline $\mathbf{F}$ & Sig & $\mathbf{T}$ & Beta & $\mathbf{B}$ & $\begin{array}{c}\text { Standard } \\
\text { Error }\end{array}$ & $\mathbf{R}^{2} \mathbf{a d}$ & $\mathbf{R}^{2}$ & $\mathbf{R}$ \\
\hline $11 / 998$ &.$/ 001$ & $3 / 464$ &.$/ 183$ & $1 / 376$ & $5 / 55$ &.$/ 031$ &.$/ 033$ &.$/ 183$ \\
\hline
\end{tabular}

Table 2 also depicts the two variable regression analysis for Independent Variables and Unethical Behavior.

Table 2: Two-Variable Regression between Independent Variables and Unethical Behavior

\begin{tabular}{|c|c|c|c|c|c|c|c|c|c|}
\hline $\mathbf{F}$ & Sig & $\mathbf{T}$ & Beta & $\mathbf{B}$ & Standard Error & $\mathbf{R}^{\mathbf{2}} \mathbf{a d}$ & $\mathbf{R}^{\mathbf{2}}$ & $\mathbf{R}$ & \\
\hline
\end{tabular}




\begin{tabular}{|c|c|c|c|c|c|c|c|c|c|}
\hline $11 / 849$ &.$/ 001$ & $3 / 442$ &.$/ 181$ &.$/ 360$ & $5 / 55$ &.$/ 03$ &.$/ 033$ &.$/ 181$ & Emotionality \\
\hline $23 / 062$ &.$/ 000$ & $4 / 802$ &.$/ 249$ &.$/ 236$ & $5 / 47$ &.$/ 559$ &.$/ 062$ &.$/ 249$ & Power \\
\hline
\end{tabular}

For the fourth and fifth hypotheses, since it was needed to measure a doubled independent variable and a distance variable, T-test was used. The scores of men and women were resulted as different, and the observed difference in mean scores indicated that the scores of men were higher than those of women. As the value of $\mathrm{T}$ and its meaningful levels of 2.244 and 0.025 were calculated, the fourth hypothesis is also confirmed.

About the fifth hypothesis, scores of men and women were different, and the difference showed the mean scores of men were higher than those of women, thus the value of $\mathrm{T}$ and meaningful level of 3.40 and 0.001 are approved. Therefore, the fifth hypothesis is also confirmed.

\section{Discussion and Conclusion}

The present study was conducted with the aim of investigating the relationship between personal characteristics of emotionality and power seeking and unethical behavior. According to the results, there is a positive significant relationship between tendency to unethical behaviors and emotionality. It means that people with higher emotionality are more expected to commit unethical behavior. These results are according to the previous researches of Zuckerman (1978), Chandra et al. (2003), and Zargar et al. (2009) which was about the unethical behavior as addiction and emotionality. Due to the tendency to variation, experience, and risk in people with high emotionality, they are more exposed to illogical methods of emotionality as unethical behaviors.

Findings of this study also showed that there is a positive significant relationship between power seeking and tendency to unethical behaviors. It can be claimed that people with higher tendency to power are more exposed to unethical behaviors, and this finding is according to researches of Law (2001) in a population of physicians, and Laguna et al. (2009) in the population of police officers.

A cause and effect relationship was also detected between emotionality and power seeking in the population which is suggestive of the fact that individuals with emotionality are more motivated for power.

Finally, based on the results indicated by hypotheses 4 and 5, men possess more emotionality and tendency to power than do their female counterparts. 


\section{References}

Adorno, T., Frenkel-Brunswike, W., E.Levinson, \& Sanford, R. (1950). The Authoriatarian Personality. New York: Harper and Row.

Atkinson, L. R. (2006). Hilgard`s Psychology (Translated by Rafeei), 5th edition ed., Vol. 2. Tehran: Arjmand Publication.

Brown, T. A., Sautter, J. A., Littvay, L., Sautter, A. C., \& Bearnes, B. (2010). Ethics and Personalit Empathy and Narcissism as Moderators of Ethical Decision Making in Business Students. Journal of Ecucation for Business , 85 (4), 203-208.

Clark, L. A. (1991). General Affective Dispositions in Physical- and Psychological Health. In R. F. Synder D. (Ed.), Handbook of Social and Clinical Psychology. New York: Permagon Press. 245-221 .

Gaudine Alice, L. T. (2001). Emotion and Ethical Decision-Making in Organizations. Journal of Business Ethics , 31 (2), 175-187.

Ghasemi, B. (2010). Organizational Behavior Management (In Persian), Vol. 1. Tehran: Sepahan Publication.

Izard, C. (1993). Four Systems for Emotion Activation, Cognitive Noncognitive Development. Psychological review, 100.

Judge, T. A. (1993). Does Affective Disposition Moderate the Relationship Between Job Satisfaction and Voluntary Turnover? Journal of Applied Psychology , 73 (3), 395-401.

Kalat, J. (2008). Physiological Psychology (Translated by Yahya Seyed Mohammadi). Tehran: Ney Publications.

Kumar C. Rallapalli, S. J. (1994). Consumer Ethical Beliefs and Personality Traits: An Exploratory Analysis. Journal of Business Ethics , 487-495.

Levine, E. L. (2010). Emotion and Power (as Social Influence): Their Impact on Organizational Citizenship and Counterproductive Individual andOorganizational Behavior. Human Resource Management Review , 7-14.

Louis Laguna, A. L. (2009). An Examination of Authoritarian Personality Traits among Police Officers: The Role of Experience. Journal of Police and Criminal Psychology , 25 (2), 99-104.

Lowe Michael, I. K. (2001). Is it Possible to Assess the "Ethics" of Medical School Applicants? Journal of Medical Ethics , 27, 404-408.

Naude, I., Anita, M. L., Richard, F., \& Jess, N. (2004). Factors Impacting on Ethical Behavior in Organizations; University of Pretoria. Surveyor"s perspectives on ethics in organization . 
Parkes, K. R. (1990). Coping, Negative Affectivity, and the Work Environment: Additive and Interactive Predictors of Mental Health. Journal of Applied sychology, 75 (4), 399-409.

Rafaeli, a. \&. (1989). The expression of emotion in organizational life. In. (B. M. L. L. Cummings, Ed.) Research in Organizational Behavior, 11, 1-43.

Rezaeeyan, A., \& Naeeji, M. J. (Modiriat Vision Magazine). Effects of Emotionality and Personality on Job Satisfaction. Modiriat Vision Magazine , 33, 49-66.

Robins, S. P. \& T. A. Judge. 2009. Organizational Behavior, $13^{\text {th }}$ Ed. Pearson: Prentice Hall.

Sackett, P. R., \& DeVore, C. J. (2001). Counterproductive Behaviors at Work. In D. S. N. Anderson (Ed.), Handbook of industrial,work, and organizational psychology,1:145-164.

Sharon, S., Naquin, E. F., \& Holton, I. (2002). The Effects of Personality,Affectivity, and Work Commitment on Motivation to Improve Work Through Learning. Human Resource Development Quarterly, 13 (4).

Shaw, J. D., Duffy, M. K., G. D., J., \& Gupta, N. (2003). Positive and Negative Affect, Signal Sensitivity and Pay Satisfaction. Journal of Management , 25: 189-197.

Shriftz, G. M., \& Out, G. S. (2009). Organization Theories (Translated by Ali Parsaeeyan), Vol. 2. Tehran: Termeh Publication.

Sidanius, J., \& Pratto, F. (1999). Social Dominance: An Intergroup Theory of Social Hierarchy and Oppression. New York: Cambridge University Press.

Spector, P. E., Zapf, D., Chen, P. Y., \& Frese, M. (2000). Don't Throw Out the Baby with the Bath Water: The Role of Negative Affectivity in Reports of Job Stressors and Strains. Journal of Organizational Behavior, 79-95.

The American Heritage ${ }^{\circledR}$ Medical Dictionary. 2007. Houghton Mifflin Company.

Weber, M. (1947). The theory Of social and economic organization. New York: Free Press.

Witt, J. C., Clark, L. A., \& Elliott, S. N. (2002). Cognitive- Affective Stress Response: Effects of Individual Stress Propensity on Physiological and Psychological Indicators of Strain. Psychological Reports , 88:768-784. 